\title{
In Vivo Transfer of Nitric Oxide between a Plasma Protein-bound Reservoir and Low Molecular Weight Thiols
}

\author{
Jonathan S. Scharfstein, ${ }^{\star}$ John F. Keaney, Jr., ${ }^{\ddagger}$ Adam Slivka, ${ }^{*}$ George N. Welch, ${ }^{\star}$ Joseph A. Vita, ${ }^{\ddagger}$ Jonathan S. Stamler, ${ }^{5}$ \\ and Joseph Loscalzo* ${ }^{\prime \prime}$ \\ ${ }^{\ddagger}$ Whitaker Cardiovascular Institute and Evans Department of Medicine, Boston University School of Medicine, Boston, Massachusetts \\ 02118; ${ }^{8}$ Duke University School of Medicine, Durham, North Carolina 27701; and *Department of Medicine, Brigham and Women's \\ Hospital, Brockton/West Roxbury V.A. Medical Center, Harvard Medical School Boston, Massachusetts 02132
}

\begin{abstract}
Plasma albumin reacts with nitric oxide (NO) to form the bioactive adduct, $S$-nitroso-albumin ( $S$-NO-albumin). The limited intracellular access of $S$-NO-albumin suggests the need for a vascular transfer mechanism of NO from a large plasma $S$-NO-albumin pool to effect biologic function. To study the role of low molecular weight (LMW) thiols in NO transfer in vivo, we administered intravenous $S$-NOalbumin (1-300 nmol/kg) to rabbits before and after an intravenous infusion of $\mathrm{L}$-cysteine or $\boldsymbol{N}$-acetyl-L-cysteine. $S$ NO-albumin produced dose-dependent hypotension that was significantly augmented by prior infusion of either LMW thiol. LMW thiol infusion significantly accelerated the rate of onset and reduced the duration of action of the hypotension induced by $S$-NO-albumin. The hemodynamic effects of $S$-NO-albumin after pretreatment with LMW thiols were mimicked by administration of the corresponding LMW $S$-nitrosothiol. The transfer of NO from albumin to L-cysteine was directly measured in rabbit plasma using a novel technique that couples high performance liquid chromatography to electrochemical detection. These data demonstrate that NO exchange between plasma protein thiolbound NO and available LMW thiol pools (transnitrosation) occurs in vivo. (J. Clin. Invest. 1994. 94:1432-1439.) Key words: nitric oxide • proteins • thiols • endotheliumderived relaxing factor $\cdot S$-nitrosothiols
\end{abstract}

\section{Introduction}

Since the discovery of endothelium-derived relaxing factor (EDRF) ${ }^{1}$ by Furchgott and Zawadzki in 1980 (1), much information has become available about the biochemistry and physiology of this endothelial product. Many investigators have

This work was presented in abstract form at the 65th Scientific Sessions of the American Heart Association, Dallas, TX, November 16-19, 1992.

Address correspondence to Joseph Loscalzo, M.D., Ph.D., Boston University Medical Center, Whitaker Cardiovascular Institute, $80 \mathrm{E}$ Concord Street, W507, Boston, MA 02118.

Received for publication 17 June 1993 and in revised form 1 July 1994.

1. Abbreviations used in this paper: EDRF, endothelium-derived relaxing factor; NO, nitric oxide.

J. Clin. Invest.

(c) The American Society for Clinical Investigation, Inc.

$0021-9738 / 94 / 10 / 1432 / 08 \quad \$ 2.00$

Volume 94, October 1994, 1432-1439 found that EDRF shares similar kinetic, biochemical, and physiologic properties with the nitric oxide radical (NO). Both agents cause equipotent relaxations of arterial and venous smooth muscle (2-4), inhibit platelet aggregation (5-8), and activate guanylyl cyclase (3-4). At least two groups have found the halflives of these two agents to be identical $(2,9)$.

Despite these extensive similarities, several investigators have reported important differences between EDRF and NO. While some investigators have observed that EDRF and NO both relax nonvascular smooth muscle $(10)$, in some studies EDRF fails to do so consistently (11). Myers et al. (12) found that EDRF was 80 -fold more potent than $\mathrm{NO}$ in a bioassay system. In addition, Kelm and Schrader (13) reported the halflife of NO in vivo to be $\sim 0.1 \mathrm{~s}$, considerably shorter than the estimated 3-30-s half-life of EDRF ex vivo $(9,12)$.

Although several of these studies have been criticized for methodologic flaws and the experimental systems used varied, taken together these data have led to the speculation that EDRF bioactivity may not be entirely accounted for by NO alone, but may be evinced by a closely related NO adduct $(12,14-15)$. One likely candidate class of molecular species that has been shown to react readily with NO under physiologic conditions, without inactivating it, is that of biologic thiols, which form thionitrites or $S$-nitrosothiols (RSNOs) (14-16). We have recently shown that the sulfhydryl group(s) of plasma proteins can combine with oxides of nitrogen under physiologic conditions to form stable, biologically active NO adducts (17-20). The physiologic relevance of such protein-associated NO has been highlighted by recent evidence that NO circulates in mammalian plasma primarily as $S$-nitroso-albumin (18) and by the demonstration that $S$-nitroso-albumin possesses EDRF-like properties in vivo $(20)$.

Although these direct plasma measurements and in vivo experimental data confirm the existence and biologic activity of $S$-nitroso-albumin, the role of serum albumin in NO metabolism remains imprecisely defined. It is possible that $\mathrm{S}$-nitroso-albumin acts as an extracellular "buffer" of NO from which NO can be transferred to the intracellular milieu by smaller, less diffusion-limited $S$-nitrosothiol species. To investigate this possibility, we studied the effects of low molecular weight thiols on the systemic hemodynamic responses to $S$-nitroso-albumin in a rabbit model. Using a novel technique that couples reversephase high performance liquid chromatography (HPLC) with electrochemical detection (ECD), we also obtained direct evidence for the biochemical transnitrosation of NO from a plasma albumin pool to a low molecular weight thiol pool in vivo.

\section{Methods}

\section{Materials}

Sulfanilamide, $N$-(1-naphthyl)ethylenediamine dihydrochloride, and $N$ acetyl-L-cysteine were purchased from Aldrich Chemical Co. (Milwau- 
kee, WI). Sodium nitrite was obtained from Fisher Scientific (Fairlawn, $\mathrm{NJ}$ ) and atropine sulfate was obtained from LyphoMed Inc. (Rosemont, IL). Xylazine was purchased from Mobay Corp. (Shawnee, KS ). Ketamine hydrochloride was obtained from Aveco Co., Inc. (Fort Dodge, IA) and sodium pentobarbital was purchased from Anthony Products Co. (Arcadia, CA). Hexamethonium bromide, timolol maleate, L-cysteine, bovine serum albumin, and all other reagent-grade chemicals were purchased from Sigma Chemical Co. (St. Louis, MO).

\section{Synthesis of $S$-nitroso-serum albumin and S-nitrosothiols}

$S$-nitroso-albumin ( $S$-NO-albumin) was prepared as previously described (17). Briefly, bovine serum albumin was exposed to an equimolar concentration of sodium nitrite in $1.0 \mathrm{~N} \mathrm{HCl}$ for $30 \mathrm{~min}$ at $25^{\circ} \mathrm{C}$ and the solution was subsequently neutralized with equal volumes of Trisbuffered saline (TBS, $10 \mathrm{mM}$ Tris, $0.15 \mathrm{M} \mathrm{NaCl}, \mathrm{pH} 7.4$ ) and $1.0 \mathrm{~N}$ $\mathrm{NaOH}$. The presence of an $S$-NO bond was confirmed and quantified by the method of Saville (21), and synthesis of $S$-nitroso-BSA by this method yielded a stoichiometry of $0.85 \pm 0.07 \mathrm{~mol} S-\mathrm{NO} / \mathrm{mol}$ BSA. Previous studies have also confirmed the presence of an $S$-nitrosothiol using $\left[{ }^{15} \mathrm{~N}\right]$ NMR spectroscopy and chemiluminescence (17).

$S$-nitroso-L-cysteine ( $S$-NO-cysteine) and $S$-nitroso- $N$-acetyl-L-cysteine ( $S$-NO- $N$-acetyl-cysteine) were synthesized by combining equimolar concentrations of L-cysteine (cysteine) or $\mathrm{N}$-acetyl-L-cysteine ( $\mathrm{N}$ acetyl-cysteine) with sodium nitrite in $0.5 \mathrm{~N} \mathrm{HCl}$ at room temperature Synthesis of $S$-NO-cysteine and $S$-NO- $N$-acetyl-cysteine by this method produces $S$-nitrosothiol that is $\geq 99 \%$ pure and stable at $\mathrm{pH} \leq 3(22)$. These low molecular weight $S$-nitrosothiols were synthesized on the day of use and maintained at $\mathrm{pH} \sim 3$ until immediately before administration, at which time they were diluted with $10 \mathrm{vol}$ TBS.

Bovine serum albumin (BSA), $N$-acetyl-L-cysteine, and L-cysteine controls were each produced by acidification and neutralization in the absence of sodium nitrite. Similarly, sodium nitrite was acidified and neutralized in the absence of BSA as another control. L-cysteine and $N$ acetyl-L-cysteine were dissolved in phosphate-buffered saline (PBS, 10 $\mathrm{mM}$ sodium phosphate, $0.15 \mathrm{M} \mathrm{NaCl}, \mathrm{pH} 7.4$ ) and titrated to a $\mathrm{pH}$ of 4. All doses used in the experiments described were based on the component of mercuric ion-displaceable NO.

\section{Measurement of plasma S-nitrosothiols}

Plasma $S$-NO-cysteine was measured by HPLC using a C18 reversephase column coupled to an electrochemical detector with a dual $\mathrm{Au} /$ $\mathrm{Hg}$ electrode set at both oxidizing $(+0.15 \mathrm{~V})$ and reducing $(-0.15 \mathrm{~V})$ potentials versus a $\mathrm{Ag} / \mathrm{AgCl}$ reference electrode (Bioanalytical Systems, West Lafayette, IN). The working electrodes were configured in series with the reducing electrode upstream. Using this configuration, $S$-nitrosothiols are detected at both electrodes-as $S$-nitrosothiols at the reducing electrode and as reduced thiol (the product of upstream reduction) at the oxidizing electrode. The mobile phase consisted of 0.1 $\mathrm{M}$ monochloroacetic acid, $0.125 \mathrm{M}$ disodium ethylenediaminetetraacetic acid (EDTA), $1.25 \mathrm{mM}$ sodium octyl sulfate, and $1 \%$ ( $\mathrm{vol} / \mathrm{vol}$ ) acetonitrile, $\mathrm{pH} 2.8$. The flow rate was $1 \mathrm{cc} / \mathrm{min}$. This technique can reliably separate and detect $S$-nitrosothiols in the nanomolar range. Blood samples were diluted 1:10 with citrate-phosphate-dextrose (CPD) anticoagulant $\left(15.6 \mathrm{mM}\right.$ citric acid, $90 \mathrm{mM}$ sodium citrate, $16 \mathrm{mM} \mathrm{NaH}_{2}$ $\mathrm{PO}_{4} \cdot \mathrm{H}_{2} \mathrm{O}$, and $142 \mathrm{mM}$ dextrose, $\left.\mathrm{pH} 7.35\right)$ and $75 \mathrm{mg} \%$ diethylenetriaminepentaacetic acid (DTPA), $\mathrm{pH} 7.4$, then immediately centrifuged at $1,000 \mathrm{~g}$ for $10 \mathrm{~min}$ at $4^{\circ} \mathrm{C}$. Plasma was microfiltered using a Centrifree microfiltration system (Amicon, Beverly, MA) with a molecular weight cutoff of $30,000 \mathrm{D}$ at $1,500 \mathrm{~g}$. The resulting filtrate was injected into the HPLC/ECD apparatus and S-nitrosothiols were identified and quantitated by comparison with authentic standards. Biochemical identity of $S$-nitrosothiol peaks was also confirmed by augmentation of peak height by the addition of authentic (internal) standard to the plasma samples.

\section{Measurement of plasma thiols}

Plasma thiols were measured by HPLC/ECD. The reduction of plasma thiols was performed by a modification of a previously described technique (23). Briefly, blood samples were diluted 1:10 with citrate-phos- phate-dextrose (CPD) anticoagulant and $75 \mathrm{mg} \%$ DTPA, pH 7.4, and immediately centrifuged at $1,000 \mathrm{~g}$ for $10 \mathrm{~min}$ at $4^{\circ} \mathrm{C}$. $150 \mu \mathrm{l}$ of plasma were then mixed with $50 \mu \mathrm{l} 0.1 \mathrm{M}$ potassium borate, $\mathrm{pH} 9.5$, containing $2 \mathrm{mM}$ ethylenediaminetetraacetic acid (EDTA). $20 \mu \mathrm{l}$ of $100 \mathrm{ml} / \mathrm{liter}$ of tri-n-butyl-phosphine was added and the sample was incubated at $4^{\circ} \mathrm{C}$ for $30 \mathrm{~min}$. The sample was then microfiltered using a Centrifree microfiltration system at $1,500 \mathrm{~g}$. The resulting filtrate was injected into the HPLC/ECD apparatus with both electrodes set at $+0.15 \mathrm{~V}$ configured in parallel, and thiols were identified and quantitated by comparison with authentic standards.

\section{Animal preparation}

New Zealand White rabbits $(3.5-5.0 \mathrm{~kg}$ ) were sedated with $0.1 \mathrm{mg} /$ $\mathrm{kg}$ atropine sulfate and $5 \mathrm{mg} / \mathrm{kg}$ xylazine intramuscularly (IM) followed by $40 \mathrm{mg} / \mathrm{kg}$ of ketamine hydrochloride. Anesthesia was maintained by intravenous infusion of sodium pentobarbital at $5-7 \mathrm{mg} / \mathrm{kg}$ per $\mathrm{h}$ with a Harvard Pump (model 22, Harvard Apparatus Co. Inc., South Natick, MA). Tracheostomies were performed through a midline neck incision, and the trachea was intubated with a specifically designed endotracheal tube. Body temperature was maintained with a homeothermic blanket system (Harvard Apparatus Ltd., Edinbridge, KY, UK).

The femoral vein was cannulated through an inguinal incision with polyethylene tubing (PE-90; Clay Adams, Parsipanny, NJ) for intravenous access, and the femoral artery was cannulated with a 5 French high fidelity pressure transducer (model SPC-350; Millar Instruments, Houston, TX). A Doppler flow probe (model T101; Transonic Systems Inc., Ithaca, NY) was placed on the contralateral femoral artery to measure hindlimb blood flow. All measurements were recorded on a Gould physiograph (model 2600; Gould Electronics Inc., Cleveland, $\mathrm{OH}$ ).

Reflex responses were minimized in rabbits in groups 1-3 with hexamethonium bromide ( $20 \mathrm{mg} / \mathrm{kg}$ intravenously over $20 \mathrm{~min}$ ), timolol maleate $(1 \mathrm{mg} / \mathrm{kg}$ intravenous bolus $)$, and atropine sulfate $(0.2$ $\mathrm{mg} / \mathrm{kg}$ intravenous bolus). Autonomic blockade was maintained with continuous infusions of hexamethonium bromide $(10 \mathrm{mg} / \mathrm{kg}$ per $\mathrm{h})$ and timolol maleate $(0.06 \mathrm{mg} / \mathrm{kg}$ per $\mathrm{h})$.

\section{Experimental protocols}

Group 1. After the establishment of a baseline hemodynamic state, rabbits received intravenous bolus doses of $S$-NO-albumin (1-300 $\mathrm{nmol} / \mathrm{kg}$ ) in random order of concentration. A new baseline steadystate was established prior to the administration of each dose. After completion of this dosage regimen, rabbits received an intravenous infusion of $613 \mu \mathrm{mol} / \mathrm{kg}$ of either L-cysteine $(n=5)$ or $N$-acetyl-L-cysteine $(n=6)$ over $20 \mathrm{~min}$ followed by repeated administration of $S$-NOalbumin at identical doses.

Group 2. Rabbits in this group were prepared as described above and received intravenous bolus doses of $S$-NO-cysteine $(n=5)$ or $S$ NO- $N$-acetyl-cysteine $(n=6)(1-300 \mathrm{nmol} / \mathrm{kg})$ in random order. These rabbits also served as their own controls, receiving similar doses of L-cysteine or $\mathrm{N}$-acetyl-L-cysteine after the initial dosage regimen was completed.

Group 3. Rabbits in this group $(n=5)$ served as controls for group 1 , and received intravenous bolus doses $(1-300 \mathrm{nmol} / \mathrm{kg})$ of BSA and sodium nitrite controls in an escalating dosage regimen.

Group 4. Rabbits in this group $(n=10)$ were used for measurement of $S$-nitrosothiols. A single $300 \mathrm{nmol} / \mathrm{kg}$ dose of $S$-NO-albumin was administered intravenously to each rabbit before and after a 10-min infusion of $400 \mu \mathrm{mol} / \mathrm{kg}$ L-cysteine. The $S$-NO-albumin used for this group of rabbits was synthesized by combining a 1.5 -fold molar excess of bovine serum albumin with sodium nitrite to minimize free sodium nitrite in the synthesized product $(\leq 50 \mu \mathrm{M})$. Blood samples were drawn for measurement of plasma $S$-NO-cysteine at baseline, as well as 1 min after administration of $S$-NO-albumin following pre-infusion of L-cysteine.

Group 5. Rabbits in this group $(n=4)$ served as controls for rabbits in group 4 and received $60 \mathrm{nmol} / \mathrm{kg}$ of acidified and neutralized sodium nitrite as an intravenous bolus both before and after a 10-min infusion 


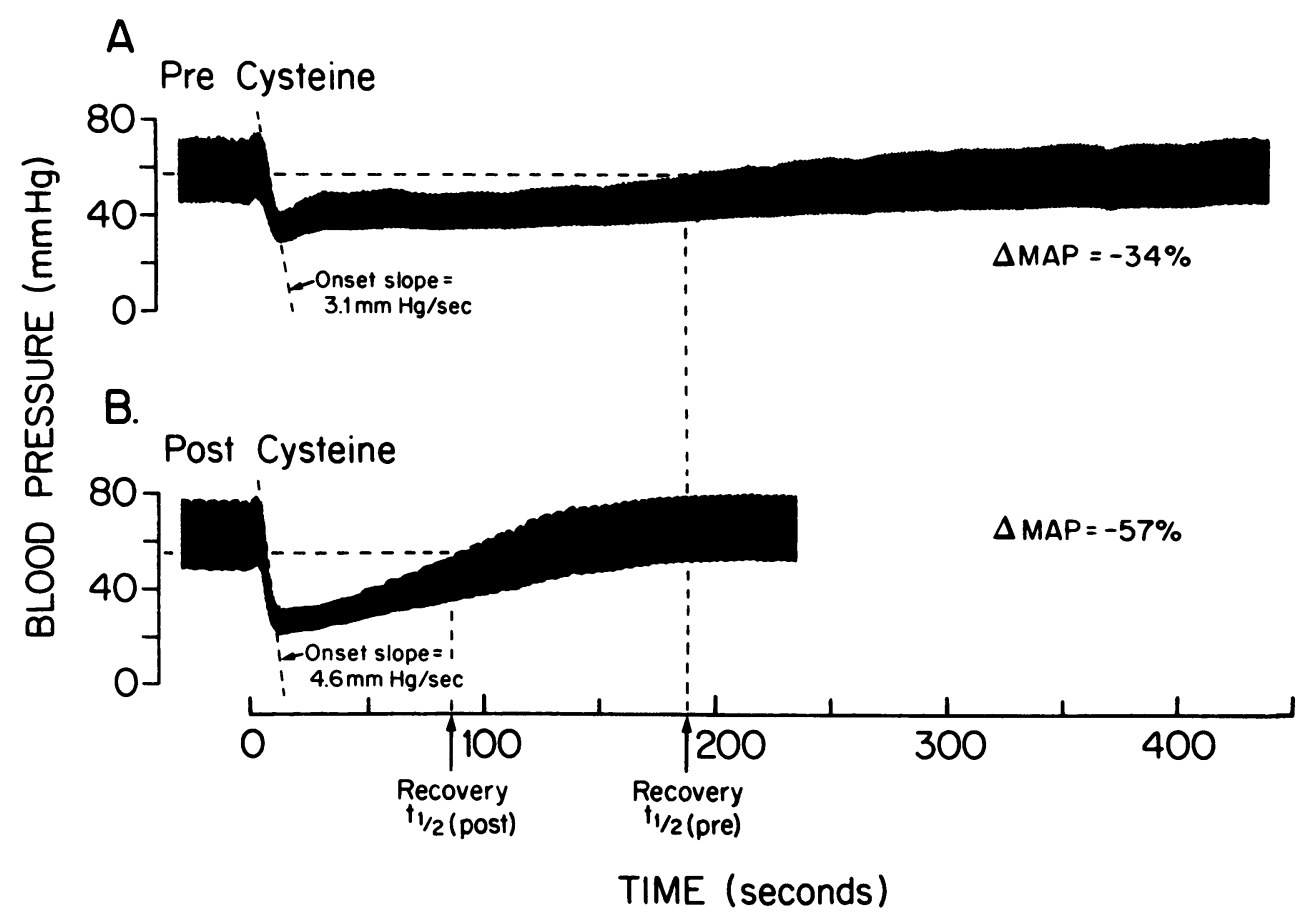

Figure 1. Sample tracing of the hemodynamic effect of $S$-NO-albumin before and after infusion of cysteine. One hundred $\mathrm{nmol} / \mathrm{kg} S$ NO-albumin $(A)$ produced a rapid fall in blood pressure followed by a gradual asymptotic return to baseline. After infusion of $\mathbf{6 1 3}$ $\mu \mathrm{mol} / \mathrm{kg}$ cysteine $(B)$, the hypotensive response induced by the same dose of $S$-NO-albumin was potentiated in magnitude by $39 \%$ $(P<0.0001)$ and accelerated by 58\% $(P<0.003)$. Similarly, the recovery half-time was reduced by $61 \%(P<0.01)$. As demonstrated in the figure, the rate of onset of the hypotensive response (onset slope) was derived from the maximal slope of the decline in the systolic arterial blood pressure tracing, and the recovery half-time ( rec. $t_{1 / 2}$ ) was calculated by measuring the time for recovery of the systolic arterial blood pressure to half its maximal decrease. of $400 \mu \mathrm{mol} / \mathrm{kg}$ L-cysteine. This dose was chosen because it reflected at least a $20 \%$ increase over the maximal dose of free sodium nitrite in the bolus of $S$-NO-albumin administered to rabbits in group 4 .

Group 6. Rabbits in this group $(n=10)$ were used for measurement of plasma L-cysteine levels. A single $300 \mathrm{nmol} / \mathrm{kg}$ dose of $S$-NO-albumin was administered intravenously to each rabbit before and after a 10 -min infusion of $613 \mu \mathrm{mol} / \mathrm{kg} \mathrm{L}$-cysteine. The $S$-NO-albumin used for this group of rabbits was prepared as described for rabbits in group 4. Blood samples were drawn for measurement of plasma L-cysteine at baseline, as well as $1 \mathrm{~min}$ after administration of $S$-NO-albumin after pre-treatment with L-cysteine.

\section{Definition of hemodynamic and time parameters}

$\mathrm{EC}_{50}$ was defined as the dose of $S$-NO-albumin that caused a halfmaximal decrease in mean arterial blood pressure. This parameter was determined for the dose-range tested and does not reflect an actual pharmacologic $\mathrm{EC}_{50}$ per se. Recovery half-time was calculated by measuring the time to recovery of the systolic arterial blood pressure to half its maximal decrease (Fig. 1). This parameter was chosen as a refiection of recovery time because of the asymptotic nature of the restoration in blood pressure after a dose of test agent. $E_{100 \mathrm{~s}}$ was defined as the dose of $S$-NO-albumin that effected a recovery time of $100 \mathrm{~s}$. Rate of onset was obtained by calculating the maximal slope of the decline of the systolic arterial blood pressure tracing (Fig. 1). $\mathrm{EC}_{-2 \mathrm{mmH} / \mathrm{s}}$ was defined as the dose of $S$-NO-albumin that effected a slope of $-2 \mathrm{~mm} \mathrm{Hg} / \mathrm{s}$. Hindlimb resistance was calculated by dividing the nadir of mean arterial pressure by the coincidental peak increase in mean hindlimb blood flow after administration of a dose of test agent and is expressed in Wood units $(\mathrm{mm} \mathrm{Hg} \cdot \mathrm{min} /$ liter $)$.

\section{Statistical analysis}

All data are presented as the mean \pm SEM. All dose-response curves were analyzed by repeated measures analysis of variance (SAS Institute, Inc., Cary, NC). Dose-response curves were compared among agents by the same method. Comparison of $S$-NO-cysteine and L-cysteine concentrations at baseline and after administration of $S$-nitroso-albumin following pre-treatment with L-cysteine was performed using a paired, one-tailed Student's $t$ test. Statistical significance was accepted if the null hypothesis was rejected with $P<0.05$.

\section{Results}

Hemodynamics: blood pressure response. A representative tracing depicting the effect of an intravenous bolus dose of $S$-NOalbumin on mean arterial pressure is shown in Fig. 1, and doseresponse curves for this variable are shown in Fig. 2. S-NOalbumin produced a significant dose-dependent decrease in mean arterial blood pressure $(P<0.0001)$. Infusion of L-cysteine (Fig. $2 A$ ) enhanced the vascular sensitivity to $S$-NOalbumin 18-fold ( $\mathrm{EC}_{50}$ before cysteine, $62.5 \mathrm{nmol} / \mathrm{kg}$; after cysteine, $3.5 \mathrm{nmol} / \mathrm{kg} ; P \leq 0.01$ ), while infusion of $N$-acetyl-Lcysteine was associated with a three-fold enhancement of the vascular sensitivity to $S$-NO-albumin $\left(\mathrm{EC}_{50}\right.$ before $\mathrm{N}$-acetylcysteine, $62.5 \mathrm{nmol} / \mathrm{kg}$; after $N$-acetyl-L-cysteine, $20 \mathrm{nmol} / \mathrm{kg}$; $P<0.02$ ).

$S$-NO-cysteine and $S$-NO- $N$-acetyl-cysteine both produced significant dose-dependent reductions in mean arterial blood pressure $(P<0.0001)$. As shown in Fig. $2 A, S$-NO-cysteine was significantly more potent, on a molar basis, than $S$-NOalbumin $\left(\mathrm{EC}_{50} 7 \mathrm{nmol} / \mathrm{kg}\right.$ vs. $\left.62.5 \mathrm{nmol} / \mathrm{kg} ; P<0.005\right)$. By contrast, after the infusion of L-cysteine (Fig. $2 A$ ), S-NOalbumin was nearly equipotent to $S$-NO-cysteine $\left(\mathrm{EC}_{50} 3.5\right.$ $\mathrm{nmol} / \mathrm{kg}$ vs. $7 \mathrm{nmol} / \mathrm{kg}, P<0.02$ ). As shown in Fig. $2 B, S$ NO- $N$-acetyl-cysteine was fourfold more potent in reducing mean arterial pressure than $S$-NO-albumin $\left(\mathrm{EC}_{50} 15 \mathrm{nmol} / \mathrm{kg}\right.$ vs. $62.5 \mathrm{nmol} / \mathrm{kg}, P<0.02$ ). After infusion of $N$-acetyl-Lcysteine, however, $S$-NO-albumin was equipotent to $S$-NO-Nacetyl-cysteine $\left(\mathrm{EC}_{50} 20 \mathrm{nmol} / \mathrm{kg}\right.$ vs. $\left.15 \mathrm{nmol} / \mathrm{kg}, P=\mathrm{NS}\right)$. L-cysteine, $N$-acetyl-L-cysteine, BSA, and sodium nitrite controls had no significant effect on systemic arterial blood pressure over this concentration range $(P<0.001$, data not shown $)$.

Kinetics of the hemodynamic response: recovery half-time. To quantify the biologic half-life of the NO adducts studied, recovery half-times (as defined in Fig. 1) were determined for each agent. Dose-response curves for this parameter were con- 


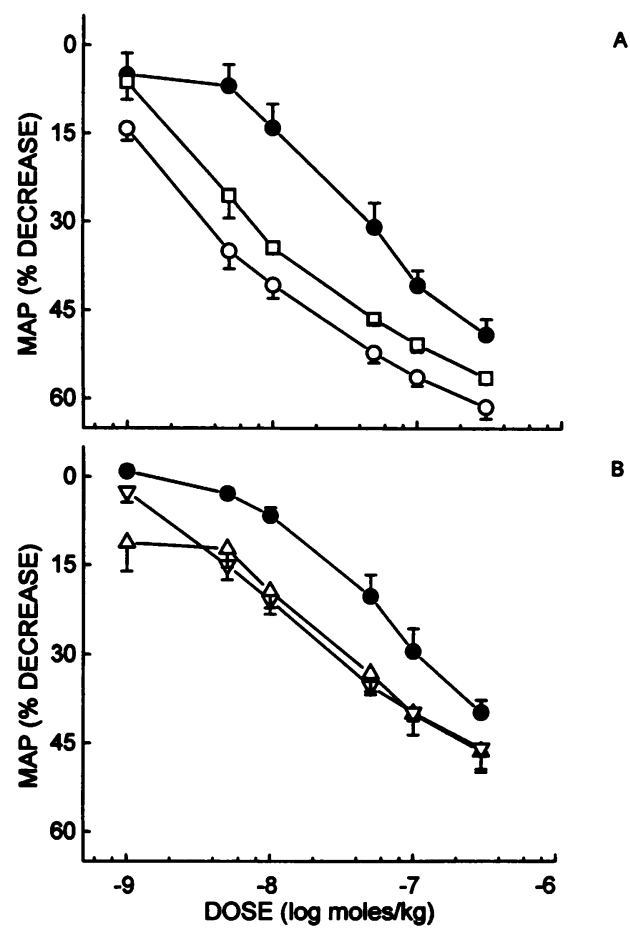

Figure 2. The systemic vascular responses to $S$-NO-albumin, $S$-nitrosocysteine, and $S$-nitroso- $N$-acetyl-cysteine. $S$-NO-albumin ( $\bullet$ ) produced a dose-dependent decrease in mean arterial pressure $(P<0.0001)$ that was significantly potentiated by infusion of $613 \mu$ moles $/ \mathrm{kg}(A)$ L-cysteine $(\mathrm{O}, P<0.001)$ and $(B) N$-acetyl-L-cysteine $(\triangle, P<0.02)$. The dose-response curve for $(A) S$-NO-cysteine ( $\square$ ) was similar to that for $S$-NO-albumin after infusion of cysteine $(P<0.02)$, and the doseresponse curve for $(B) S$-NO- $N$-acetyl-cysteine $(\nabla)$ was not significantly different from that for $S$-NO-albumin after infusion of $N$-acetylcysteine $(P=\mathrm{NS})$.

structed and are shown in Fig. 3. $S$-NO-cysteine, $S$-NO- $N$-acetyl-cysteine, and $S$-NO-albumin before and after infusion of either biologic low molecular weight thiol all produced dosedependent increases in the time for recovery of the hypotensive response $(P<0.03$ for all dose-response curves $)$. Infusion of L-cysteine produced a 10 -fold increase in the $\mathrm{EC}_{100 \mathrm{~s}}$ for $S$-NOalbumin $(18 \mathrm{nmol} / \mathrm{kg}$ vs. $180 \mathrm{nmol} / \mathrm{kg}, P<0.01)$, reflecting a significant reduction in the duration of action of $S$-NO-albumin (Fig. $3 \mathrm{~A}$ ). At a dose of $100 \mathrm{nmol} / \mathrm{kg}$ (Fig. 1), L-cysteine pretreatment reduced the recovery half-time of $S$-NO-albumin by $61 \%$. $S$-NO-cysteine administration produced recovery halftimes similar to those for $S$-NO-albumin after infusion of $\mathrm{L}$ cysteine $(P=\mathrm{NS})$.

In contrast, $\mathrm{N}$-acetyl-L-cysteine pretreatment did not produce a significant reduction in the recovery half-time or increase in $\mathrm{EC}_{100 \text { s }}$ for $S$-NO-albumin (Fig. $3 B, P=N S$ ). In concordance with this finding, the duration of action of $S$-NO- $N$-acetyl-cysteine is also similar to that for $S$-NO-albumin ( $P=\mathrm{NS})$. In addition, comparison of Fig. $3, A$ and $B$ reveals that $S$-NOcysteine has a significantly shorter duration of action than $S$ NO- $N$-acetyl-cysteine $\left(E_{100 ~ s} 275 \mathrm{nmol} / \mathrm{kg}\right.$ vs. $20 \mathrm{nmol} / \mathrm{kg}, P$ $<0.03$ ).

Kinetics of the hemodynamic response: rate of onset. The rate of onset of the hypotensive response induced by the NO adducts studied reflects the rate of delivery of NO to vascular smooth muscle. This parameter increased in a dose-dependent

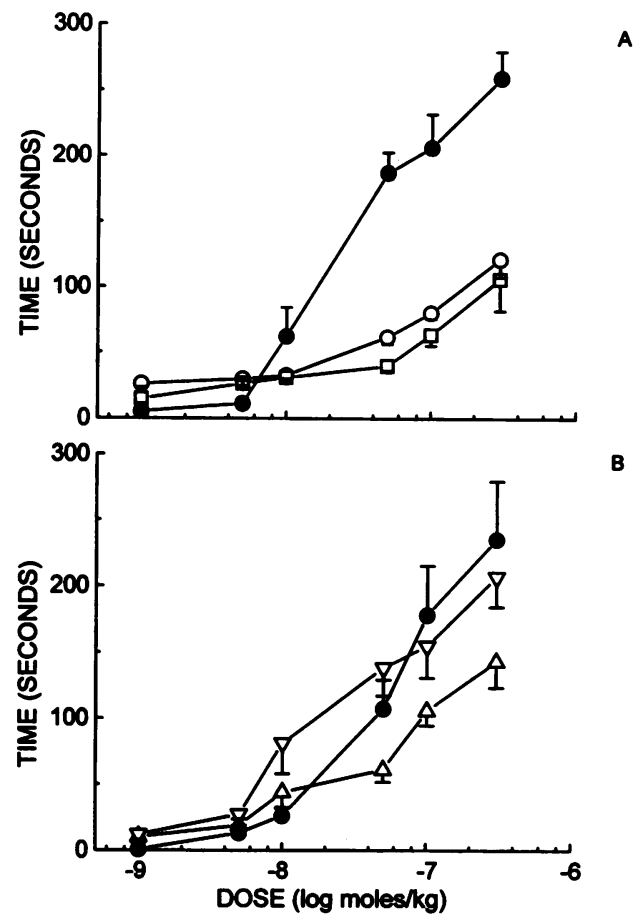

Figure 3. The duration of action of $S$-NO-albumin, $S$-NO-cysteine, and $S$-NO- $N$-acetyl-cysteine. $(A) S$-NO-albumin $(\bullet)$ produced a dose-dependent increase in the recovery half-time $(P<0.0001)$ that was significantly shortened by L-cysteine $(O, P<0.01)$. The dose-response curve for $S$-NO-cysteine ( $\square$ ) was similar to that for $S$-NO-albumin after L-cysteine pretreatment $(P=\mathrm{NS})$. ( $B$ ) $N$-acetyl-L-cysteine $(\Delta)$ did not shorten the duration of action of $S$-NO-albumin $(P=\mathrm{NS})$, and the duration of action of $S$-NO- $N$-acetyl-cysteine $(\nabla)$ was similar to that of $S$-NO-albumin $(P=\mathrm{NS})$.

fashion for both low molecular weight $S$-nitrosothiols as well as for $S$-NO-albumin before and after infusion of either lowmolecular-weight thiol (Fig. 4; $P<0.02$ for all dose-response curves). L-cysteine (Fig. $4 \mathrm{~A}$ ) accelerated the rate of onset of the hypotensive response of $S$-NO-albumin twenty seven-fold $\left(\mathrm{EC}_{-2 \mathrm{mmHg} / \mathrm{s}}\right.$ for $S$-NO-albumin before L-cysteine, $80 \mathrm{nmol} / \mathrm{kg}$; after L-cysteine, $3 \mathrm{nmol} / \mathrm{kg} ; P<0.003$ ), while $N$-acetyl-Lcysteine (Fig. $4 \mathrm{~B}$ ) infusion was associated with a four-fold decrease in the $\mathrm{EC}_{-2} \mathrm{mmHg} / \mathrm{s}(80 \mathrm{nmol} / \mathrm{kg}$ vs. $25 \mathrm{nmol} / \mathrm{kg}, P$ $<0.02$ ). At a dose of $100 \mathrm{nmol} / \mathrm{kg}, \mathrm{L}$-cysteine (Fig. 1) and $N$ acetyl-L-cysteine infusions significantly accelerated the rate of onset of hypotension by 58 and $49 \%$, respectively.

With regard to the low molecular weight $S$-nitrosothiols, $S$ NO-cysteine (Fig. $4 \mathrm{~A}$ ) and $S$-NO- $N$-acetyl-cysteine (Fig. 4 $B$ ) were both significantly more potent, on a molar basis, in accelerating the rate of onset of the hypotensive response compared to $S$-NO-albumin before infusion of thiol. $S$-NO-cysteine (Fig. $4 A$ ) was eight-fold more potent $\left(\mathrm{EC}_{-2 \mathrm{mmHg} / \mathrm{s}}, 10 \mathrm{nmol} /\right.$ $\mathrm{kg}$ vs. $80 \mathrm{nmol} / \mathrm{kg}, P<0.04$ ), and $S$-NO- $N$-acetyl-cysteine was fourfold more potent than $S$-NO-albumin $\left(\mathrm{EC}_{-2 \mathrm{mmHg}}, 20\right.$ $\mathrm{nmol} / \mathrm{kg}$ vs. $80 \mathrm{nmol} / \mathrm{kg}, P<0.03)$. $S$-NO-cysteine and $S$ $\mathrm{NO}-\mathrm{N}$-acetyl-cysteine produced rates of onset that were similar to those produced by $S$-NO-albumin after pretreatment with the corresponding low molecular weight thiol $(P=\mathrm{NS})$.

Hindlimb vascular resistance. Administration of systemic intravenous $S$-NO-albumin before and after administration of low-molecular-weight thiols produced a significant dose-depen- 


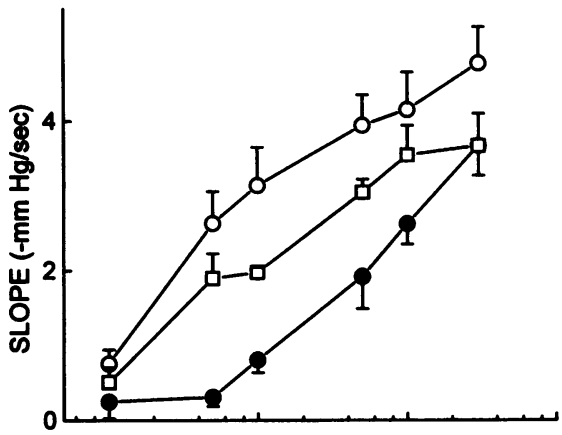

A



Figure 4. The rate of onset of the hypotensive effect of $S$-NO-albumin, $S$-NO-cysteine, and $S$-NO- $N$-acetyl-cysteine. $S$-NO-albumin $(\bullet)$ caused a dose-dependent increase in the rate of onset of the hypotensive response $(P<0.0001)$ that was significantly enhanced by prior infusion of $(A)$ L-cysteine $(O, P<0.002)$ and $(B) N$-acetyl-L-cysteine $(\triangle, P$ $<0.02)$. The dose-response curves for $S$-NO-cysteine $(\square)$ and $S$-NO$N$-acetyl-cysteine $(\nabla)$ were not significantly different from the doseresponse curves induced by $S$-NO-albumin after infusion of L-cysteine and $N$-acetyl-L-cysteine, respectively $(P=\mathrm{NS})$.

dent decrease in hindlimb resistance (Fig. 5, $P<0.03$ for all curves). Infusion of L-cysteine (Fig. $5 \mathrm{~A}$ ) significantly potentiated the decrease in hindlimb resistance induced by $S$-NO-albu$\min (P<0.05) . N$-acetyl-L-cysteine (Fig. $5 B$ ) also tended to potentiate the fall in hindlimb resistance, although this potentiation was not as marked $(P=0.07)$. Of interest, $N$-acetyl-Lcysteine infusion alone caused a significant increase in hindlimb blood flow (data not shown), and this effect may account, in part, for the less dramatic enhancement of the effects of $S$-NOalbumin on hindlimb resistance after infusion of this thiol.

Synthetic $S$-NO-cysteine and $S$-NO- $N$-acetyl-cysteine also produced significant dose-dependent decreases in hindlimb resistance $(P<0.02)$. The dose-response curves for synthetic low molecular weight $S$-nitrosothiols mimicked the dose-response curves for $S$-NO-albumin after infusion of the corresponding low molecular weight thiol ( $P=\mathrm{NS}$ for both comparisons).

Plasma S-NO-cysteine measurements. The results of $S$-NOcysteine measurements in plasma for rabbits in group 4 are shown in Fig. 6, and a representative chromatogram is shown in Fig. 7. Importantly, $S$-NO-cysteine was detected in 7 of 10 rabbits in the baseline state. The range was $30.1 \mathrm{nM}$ to $873 \mathrm{nM}$ with a mean $S$-NO-cysteine concentration of $221.1 \mathrm{nM} \pm 259.7$ $\mathrm{nM}$. Administration of $300 \mathrm{nmol} / \mathrm{kg} S$-NO-albumin following infusion of L-cysteine caused an increased in $S$-NO-cysteine concentration to $2.75 \pm 2.42 \mu \mathrm{M}$ reflecting an twelve-fold increase over baseline concentration $(P=0.017)$. Administration

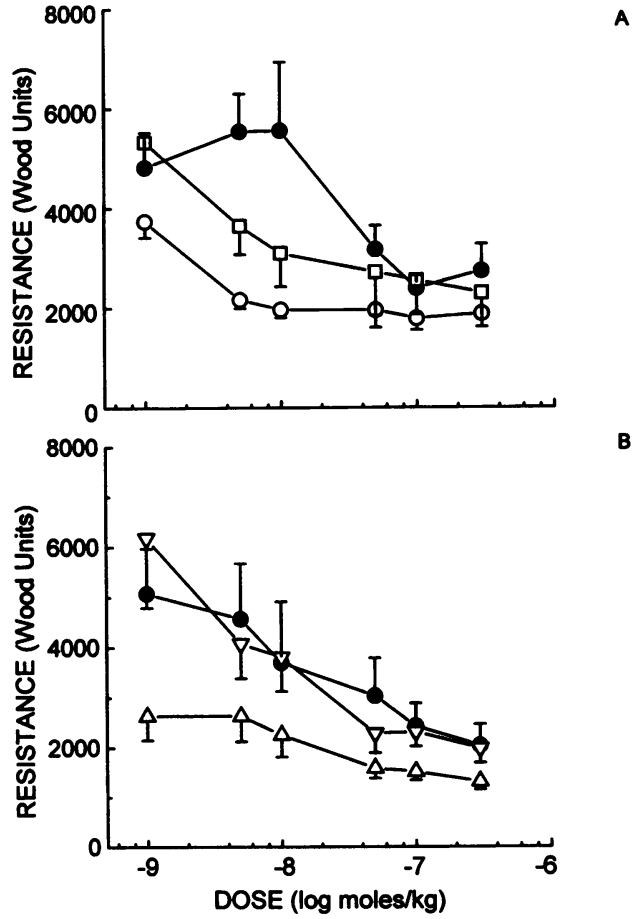

Figure 5. The response of hindlimb vascular resistance to $S$-NO-albumin, $S$-NO-cysteine, and $S$-NO- $N$-acetyl-cysteine. $S$-NO-albumin $(\bullet)$ produced a dose-dependent decrease in hindlimb vascular resistance that was significantly potentiated by L-cysteine $(O, P<0.05)$ and enhanced by $N$-acetyl-L-cysteine $(\triangle, P=0.07)$. The dose-response curves for $S$ NO-cysteine $(\square)$ and $S$-NO- $N$-acetyl-cysteine $(\nabla)$ were not significantly different from those for $S$-NO-albumin before or after L-cysteine and $N$-acetyl-L-cysteine, respectively $(P=\mathrm{NS})$.

of free sodium nitrite before and after L-cysteine infusion had no effect on baseline $S$-NO-cysteine concentration (data not shown). Mean plasma L-cysteine concentration increased 4.7fold $(n=10)$ after infusion of L-cysteine followed by $S$-NOalbumin $(P=0.0005)$.

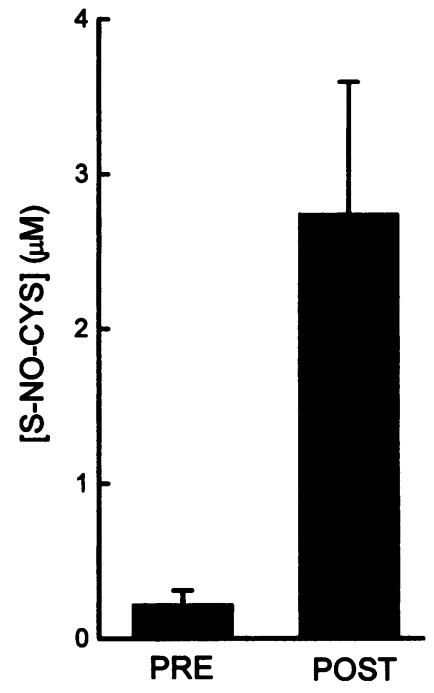

Figure 6. Measurement of $S$-nitroso-L-cysteine ( $S$-NO-CYS) concentration in vivo by HPLC coupled to ECD. Administration of $300 \mathrm{nmol} / \mathrm{kg}$ of $S$-nitroso-albumin after pretreatment with $400 \mu \mathrm{mol} / \mathrm{kg}$ cysteine (post) produced a significant 12 -fold increase in $S$-NO-cysteine ( $S$ NO-CYS) concentration compared with baseline (post) $(P$ $<0.05$ ). 


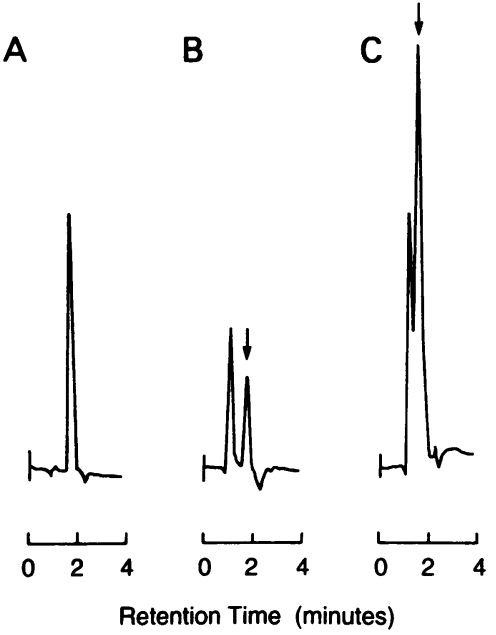

\section{Discussion}

Although the origin of NO has been definitively traced to the terminal guanidino group of L-arginine (24), the specific biochemical intermediates that exist between this precursor and the presumed site of NO action, the heme moiety of guanylyl cyclase, are unknown. Given the extreme lability and facile inactivation of NO by heme iron, non-heme iron (25), superoxide anion (26), oxygen (27), and other biochemical species (2829 ), it is likely that some intermediate molecular species serves to stabilize NO and/or transfer it from its site of origin (endothelial cell) to target effector sites (e.g., vascular smooth muscle, platelets). Plasma thiols represent likely candidates for this role as they readily form thionitrites ( $S$-nitrosothiols) under physiologic conditions by complexation with NO or by reacting with an endogenous nitrosating species $(15-16,30)$, are present in abundant quantities in vivo, and confer stability to the NO radical (14). Furthermore, there is ample evidence that low molecular weight $S$-nitrosothiols (as well as plasma protein thiols ) participate in the mechanism of action of nitrosovasodilators (14-15, 31-34), and, by inference, in the mechanism of action of NO.

We have recently shown that the $S$-nitrosation of albumin at its single free thiol, cysteine 34 , forms the bioactive NO adduct $S$-NO-albumin. $S$-NO-albumin relaxes vascular smooth muscle ex vivo (17) and in vivo (20), inhibits platelet aggregation in vitro (17) and in vivo $(20,34)$, and activates guanylyl cyclase (17). The role of $S$-NO-albumin, present in the micromolar range in mammalian plasma (17), in the intermediary metabolism of NO is, however, unclear. With prolonged in vivo hemodynamic effects (20) and limited (if any) intracellular access, $S$-NO-albumin would be unlikely to serve as an adduct that "presents" NO directly to the intracellular milieu. However, $S$-NO-albumin may serve as an extracellular buffer or reservoir of relatively stable NO that can be transferred more efficiently to vascular smooth muscle or to the platelet cytosol by low molecular weight, less diffusion-limited thiol species.

NO exchange among thiols has been documented by several investigators, including Oae and coworkers (16) and others (35-37), under non-physiologic conditions. We have recently reported that transnitrosation between $S$-NO-albumin and low molecular weight thiols occurs rapidly and completely in vitro under physiologic conditions (18). In addition, we have re- cently found that NO appears to shift transiently from high molecular-weight to low molecular-weight $S$-nitrosothiol pools in vivo in response to the administration of $\mathrm{N}^{\mathrm{G}}$-monomethyl-Larginine (18).

The hemodynamic data presented here provide direct evidence that transnitrosation occurs in vivo. The hemodynamic responses to $S$-NO-albumin after infusion of L-cysteine or $\mathrm{N}$ acetyl-L-cysteine were mimicked by administration of the corresponding synthetic $S$-nitrosothiol. This similarity was particularly striking for $N$-acetyl-L-cysteine (Fig. $2 B$ ), with nearly identical dose-response curves characterizing the hemodynamic responses to both $S$-NO- $N$-acetyl-cysteine as well as $S$-NOalbumin after infusion of $\mathrm{N}$-acetyl-L-cysteine.

The kinetic data are also consistent with in vivo transnitrosation. Both L-cysteine and $N$-acetyl-L-cysteine accelerate the rate of onset of the hypotensive response to $S$-NO-albumin. For both low molecular weight thiols, the dose-response curves for this variable induced by $S$-NO-albumin after infusion of thiol were not significantly different from the dose-response curves for the corresponding synthetic $S$-nitrosothiol. These results reflect the more efficient and rapid delivery of NO to the vascular smooth muscle cell by the low molecular weight $S$-nitrosothiols formed by transnitrosation of NO from $S$-NO-albumin to the exogenously administered low molecular weight thiol.

The data for recovery half-time also support the hypothesis that transnitrosation occurs in vivo, and highlight the differences in the stability of various low molecular weight $S$-nitrosothiols. L-cysteine shortens the duration of action of $S$-NO-albumin, and the dose-response curve for $S$-NO-albumin after infusion of $\mathrm{L}$ cysteine was nearly identical to that for synthetic $S$-NO-cysteine. Although $N$-acetyl-L-cysteine infusion did not shorten the duration of action of $S$-NO-albumin, this finding was similarly consistent with the occurrence in vivo of transnitrosation as the duration of action of synthetic $S$-NO- $N$-acetyl-cysteine was also similar to that for $S$-NO-albumin.

The hindlimb vascular resistance data also provide important insights into the differences among $S$-nitrosothiols. While infusion of $\mathrm{N}$-acetyl-L-cysteine produced a significant increase in baseline mean hindlimb blood flow (with no change in hindlimb resistance), infusion of L-cysteine did not (data not shown). This increase in baseline flow induced by $N$-acetyl-Lcysteine likely results from the formation of an appreciable pool of $S$-NO- $N$-acetyl-cysteine by transnitrosation of NO from endogenous and exogenously administered $S$-NO-albumin to $N$ acetyl-L-cysteine. Although such an $S$-nitrosothiol pool is likely formed after infusion of cysteine as well, the duration of action of $S$-NO-N-acetyl-cysteine is sufficiently prolonged in the hindlimb microcirculation that baseline hindlimb blood flow is significantly increased only by $\mathrm{N}$-acetyl-L-cysteine. This rationale may also explain the lack of a statistically significant decrease in hindlimb resistance induced by $S$-NO-albumin after administration of $\mathrm{N}$-acetyl-L-cysteine. Repeat administration of $S$-NOalbumin in the presence of an appreciable, relatively long-acting $S$-NO- $N$-acetyl-cysteine plasma pool does little to decrease the already near maximally dilated hindlimb microcirculation.

Taken together, the similarities between the hemodynamic properties of $S$-NO-albumin after pretreatment with thiol to the hemodynamic profiles of corresponding $S$-nitrosothiols are convincing evidence for transnitrosation. The HPLC/ECD data provide direct biochemical confirmation of this process. Using this technique, we have detected $S$-NO-cysteine in plasma in 7 out of 10 rabbits at baseline and markedly increased levels in all 
rabbits after administration of $S$-NO-albumin following preinfusion of L-cysteine. The range of $S$-NO-cysteine in the basal state varied from low $\mathrm{nM}$ to several hundred $\mathrm{nM}$; this range was even more variable (several hundred $\mathrm{nM}$ to low $\mu \mathrm{M}$ ) after infusion of $S$-NO-albumin and cysteine. This dramatic variability is not unexpected given the highly reactive nature of $S$ nitrosothiols in biologic systems and the fact that wide variability in nitrite and nitrate levels, other labile molecular species sensitive to redox conditions, have also been reported (38).

Based on these in vivo data and our in vitro observations, we propose a hypothetical mechanism for the transnitrosation of NO among available thiol species. The cysteine at position 34 of albumin may react with $\mathrm{NO}^{+}$, nitrosonium, to form $S$ NO-albumin, the dominant form of NO in plasma. This reaction may be favored because of the anomalously low $\mathrm{pK}_{\mathrm{a}}(<5)$ of albumin's cysteine 34 , facilitating the formation of thiolate anion $\left(\mathrm{RS}^{-}\right)$at physiologic $\mathrm{pH}$ and redox modulation of $\mathrm{NO}$ (39). We postulate that $S$-NO-albumin may serve as a buffer and reservoir of NO in plasma from which NO can be transferred more efficiently to the intracellular milieu by transnitrosation to low molecular weight thiols forming the corresponding low molecular weight $S$-nitrosothiol. These latter species may be readily transported across cell membranes by thiol transport mechanisms.

In these experiments, we have facilitated this proposed transnitrosation process by adding a significant excess of low molecular weight thiol to a supplemented $S$-NO-albumin plasma pool. In the case of L-cysteine, an endogenous biologic thiol present in the micromolar range in mammalian plasma (40), administration of exogenous thiol augments L-cysteine concentrations nearly fivefold and likely alters the equilibrium balance of endogenous NO, L-cysteine, and $S$-NO-albumin. Administration of L-cysteine without $S$-NO-albumin supplementation had no hemodynamic effect in these animals; this is likely a consequence of the complex equilibria that exist among the many competing reactions with which L-cysteine is involved in vivo, such as disulfide formation with cys 34 of albumin. After $S$ NO-albumin supplementation, however, the demonstrated increase in plasma $S$-NO-cysteine concentration by exogenous Lcysteine administration results in enhanced delivery of NO to the vasculature. This is manifested by acceleration of the rate of onset, potentiation of the hemodynamic effect, and reduction of the duration of action of exogenously administered $S$-NOalbumin.

The transnitrosation of NO from albumin to biologic thiols depends on the relative reactivity of the competing high and low molecular weight thiols as well as the relative stability of the $S$-nitrosothiols formed. Factors that likely contribute to the relative reactivities of the various thiols include the $\mathrm{pK}_{\mathrm{a}} \mathrm{s}$ of the individual thiol moieties which influence nucleophilicity, the molecular structure of the individual thiols which influence thiol or thiolate reactivity, and the ambient physiologic conditions of $\mathrm{pH}$ and ionic strength (40). The marked difference between the $\mathrm{pK}_{\mathrm{a}}$ of albumin's cysteine $34\left(\mathrm{pK}_{\mathrm{a}}<5\right)(41)$ compared with the $\mathrm{pK}_{\mathrm{a}}$ of the low molecular weight biologic thiols $\left(\mathrm{pK}_{\mathrm{a}}=7-\right.$ $8)$ (40) is one important factor that should favor the transnitrosation of NO from albumin to low molecular weight thiol under physiologic conditions by facilitating thiol- $S$-nitrosothiol exchange.

Once transnitrosation has occurred and $S$-nitrosothiols have been formed, these smaller, less diffusion-limited NO adducts can transport NO more efficiently to the vascular smooth muscle cell or platelet surface. Once at the cell surface, the specific metabolic steps that lead to the activation of intracellular guanylyl cyclase and other target effector sites remain unclear. Possible mechanisms include the direct transport of $S$-nitrosothiols into the cystosol or, alternatively, cell surface interactions with membrane bound species bearing thiols or heme-containing prosthetic groups (42). This contention draws support from the findings of Malinski and Taha (43), who used a porphyrinicbased microsensor to measure single cell NO. These investigators found that a significant NO gradient exists across the vascular smooth muscle cell and platelet membrane, suggesting that transmembrane NO transport is not a diffusion-limited process.

The data presented here strongly support the occurrence of transnitrosation reactions in vivo and a role for these reactions in the transport and metabolism of NO. Alterations in transnitrosation may be an important factor in the pathophysiology of vascular disease.

\section{Acknowledgments}

This work was supported in part by National Institutes of Health grants HL40411, HL48743, HL53919, and T32 HL07604; and a Merit Review Award from the Veteran's Administration. John F. Keaney, Jr. is the recipient of a National Research Service Award (1F 32HL0063501) from the National Institutes of Health; Jonathan Stamler is the recipient of a Clinical Investigator Award from the National Institutes of Health (KO8HL02582); Joseph Vita is the recipient of a Clinician Scientist Award from the American Heart Association (91004100); and Joseph Loscalzo is the recipient of a Research Career Development Award from the National Institutes of Health (K04HL02273).

\section{References}

1. Furchgott, R. F., and J. V. Zawadzki. 1980. The obligatory role of endothelial cells in the relaxation of arterial smooth muscle by acetylcholine. Nature (Lond.). 288:373-376.

2. Palmer, R. M. J., A. G. Ferrige, and S. Moncada. 1987. Nitric oxide release accounts for the biologic activity of endothelium-derived relaxing factor. Nature (Lond.). 327:524-526.

3. Furchgott, R. F. 1983. Role of endothelium in responses of vascular smooth muscle. Circ. Res. 35:557-573.

4. Ignarro, L. J., R. E. Byrns, G. M. Buga, and K. S. Wood. 1987. Endotheliumderived relaxing factor from pulmonary artery and vein possesses pharmacologic and chemical properties identical to those of nitric oxide radical. Circ. Res. 61:866-879.

5. Mellion, B. T., L. J. Ignarro, E. H. Ohlstein, E. G. Pontecorvo, A. L. Hyman, and P. J. Kadowitz. 1981. Evidence for the inhibitory role of guanosine $3^{\prime}, 5^{\prime}$-monophosphate in ADP-induced human platelet aggregation in the presence of nitric oxide and related vasodilators. Blood. 57:946-955.

6. Radomski, R. A., R. M. J. Palmer, and S. Moncada. 1987. Comparative physiology of endothelium-derived relaxing factor, nitric oxide, and prostacyclin in platelets. Br. J. Pharmacol. 92:181-187.

7. Radomski, M. W., R. M. J. Palmer, and S. Moncada. 1986. Endogenous nitric oxide inhibits human platelet adhesion to vascular endothelium. Lancet. 2:1057-1058.

8. Radomski, M. W., R. M. K. Palmer, and S. Moncada. 1987. The role of nitric oxide and cGMP in platelet adhesion to vascular endothelium. Biochem. Biophys. Res. Commun. 148:1482-1489.

9. Ignarro, L. J., G. M. Buga, K. S. Wood, R. E. Byrns, and G. Chaudhuri. 1987. Endothelium-derived relaxing factor produced and released from artery and vein is nitric oxide. Proc. Natl. Acad. Sci. USA. 84:9265-9269.

10. Buga, G. M., M. E. Gold, K. S. Wood, G. Chaudhuri, and L. J. Ignarro 1989. Endothelium-derived nitric oxide relaxes nonvascular smooth muscle. Eur. J. Pharmacol. 161:61-72.

11. Shikano, K., C. J. Long, E. H. Ohlstein, and B. A. Berkowitz. 1988 Comparative pharmacology of endothelium-derived relaxing factor and nitric oxide. J. Pharm. Exp. Ther. 247:873-881.

12. Myers, P. R., R. L. Minor, Jr., R. Guerra, Jr., J. N. Bates, and D. G. Harrison. 1990. Vasorelaxant properties of the endothelium-derived relaxing factor more closely resemble S-nitrosocysteine than nitric oxide. Nature (Lond.). 345:161-163. 
13. Kelm, M., and J. Schrader. 1990. Control of coronary vascular tone by nitric oxide. Circ. Res. 66:1561-1575.

14. Ignarro, L. J., H. Lipton, J. C. Edwards, W. H. Barricos, A. L. Hyman, P. J. Kadowitz, and C. A. Greutter. 1981. Mechanism of vascular smooth muscle relaxation by organic nitrates, sodium nitroprusside, and nitric oxide: evidence for the involvement of $S$-nitrosothiols as active intermediates. J. Pharmacol. Exp. Ther. 218:739-749.

15. Ignarro, L. J., J. C. Edwards, D. Y. Greutter, B. K. Barry, and C. A Greutter. 1980. Possible involvement of S-nitrosothiols in the activation of guanylate cyclase by nitroso compounds. FEBS (Fed. Eur. Biochem. Soc.) Lett. 11:275278.

16. Oae, S., Y. H. Kim, D. Fukushima, and K. Shinhama. 1978. New synthesis of thionitrites and their chemical reactivities. J. Chem. Soc. Perkin Trans. I. 913917.

17. Stamler, J. S., D. I. Simon, J. A. Osborne, M. E. Mullins, O. Jaraki, D. J. Singel, and J. Loscalzo. 1991. S-nitrosylation of proteins by nitric oxide: synthesis and characterization of novel biologically active compounds. Proc. Natl. Acad. Sci. USA. 89:444-448.

18. Stamler, J. S., O. A. Jaraki, J. A. Osborne, D. I. Simon, J. F. Keaney, D. J. Singel, C. R. Valeri, and J. Loscalzo. 1992. Nitric oxide circulates in mammalian plasma primarily as an S-nitroso adduct of serum albumin. Proc. Natl. Acad. Sci. USA. 89:7674-7677.

19. Stamler, J. S., D. I. Simon, O. Jaraki, J. A. Osborne, S. Francis, M. E. Mullins, D. J. Singel, and J. Loscalzo. 1992. S-nitrosylation of tissue-type plasminogen activator confers vasodilatory and antiplatelet properties on the enzyme. Proc. Natl. Acad. Sci. USA. 89:8087-8091.

20. Keaney, J. F., D. I. Simon, J. S. Stamler, O. Jaraki, J. Scharfstein, J. A. Vita, and J. Loscalzo. 1993. NO forms an adduct with serum albumin that has endothelium-derived relaxing factor-like properties. J. Clin. Invest. 91:15821589.

21. Saville, B. 1958. A scheme for the colorimetric determination of microgram amounts of thiols. Analyst. 83:670-672.

22. Byler, D. M., D. K. Gosser, and H. Susi. 1983. Spectroscopic estimation of the extent of S-nitrosothiol formation by nitrite action on sulfhydryl groups. J. Agric. Food Chem. 31:523-527.

23. Vester, B., and K. Rasmussen. 1991. High performance liquid chromatography method for the rapid and accurate determination of homocysteine in plasma and serum. Eur. J. Clin. Chem. Clin. Biochem. 29:549-554.

24. Palmer, R. M. J., D. S. Ashton, and S. Moncada. 1988. Vascular endothelia cells synthesize nitric oxide from L-arginine. Nature (Lond.). 333:664-666.

25. Lancaster, J. R., Jr., and J. B. Hibbs, Jr. 1990. EPR demonstration of ironnitrosyl complex formation by cytotoxic activated macrophages. Proc. Natl. Acad. Sci. USA. 87:1223-1227.

26. Saran, M., C. Michel, and W. Bors. 1990. Reaction of NO with $\mathrm{O}_{2}$. Implications for the reaction of endothelium-derived relaxing factor (EDRF). Free Rad. Res. Comms. 10:221-226.

27. Bunton, C. A., D. R. Llewellyn, and G. Stedman. 1957. In Recent Aspects of the Inorganic Chemistry of Nitrogen. Special Publication No. 10, The Chemical Society, London. 113-120.
28. Downes, M. J., M. W. Edwards, T. S. Elsey, and C. L. Walters. 1976 Determination of a non-volatile nitrosamine by using denitrosylation and a chemiluminescence analyzer. Analyst. 101:742-748.

29. Pryor, W. A., and J. W. Lightsey. 1981. Mechanisms of nitrogen dioxide reactions: initiation of lipid peroxidation and the production of nitrous acid. Science (Wash. DC). 14:435-437.

30. Stamler, J. S., and J. Loscalzo. 1990. Nitrosylation of homocysteine as a novel antithrombotic mechanism. Clin. Res. 38:246a

31. Ignarro, L. J., and C. A. Gruetter. 1980. Requirement of thiols for activation of coronary arterial guanylate cyclase by glyceryl trinitrate and sodium nitrite. Biochim. Biophys. Acta. 631:221-231.

32. Gruetter, C. A., D. Y. Gruetter, J. E. Lyon, P. J. Kadowitz, and L. J. Ignarro. 1981. Relationship between cyclic guanosine $3^{\prime}: 5^{\prime}$-monophosphate formation and relaxation of coronary arterial smooth muscle by glyceryl trinitrate, nitroprusside, nitrite and nitric oxide: effects of methylene blue and methemoglobin. J. Pharmacol. Exp. Ther. 219:181-186.

33. Fung, H.-L., S. Chong, E. Kowaluk, K. Hough, and M. Kakemi. 1988 Mechanism for the pharmacologic interaction of organic nitrates with thiols Existence of an extracellular pathway for the reversal of nitrate vascular tolerance by N-acetylcysteine. J. Pharmacol. Exp. Ther. 245:524-530.

34. Chung, S., and H.-L. Fung. 1990. Thiol mediated catalysis of nitroglycerin degradation by serum proteins. Increase in metabolism was not accompanied by S-nitrosothiol production. Drug Metab. Dispos. 18:61-67.

35. Keaney, J. F., J. S. Stamler, J. Scharfstein, J. D. Folts, and J. Loscalzo. 1992. NO forms a stable adduct with serum albumin that has potent antiplatelet properties in vivo. Clin. Res. 40:194A.

36. Keen, J. H., W. H. Habig, and W. B. Jakoby. 1976. Mechanism of the several activities of the glutathione S-transferases. J. Biol. Chem. 251:61836188 .

37. Bennett, B. M., S. M. Kobus, J. F. Brien, K. Nakatsu, and G. S. Marks 1987. Requirement for reduced, unliganded hemoprotein for the hemoglobin- and myoglobin-mediated biotransformation of glyceryl trinitrate. J. Pharmacol. Exp. Ther. 237:629-635.

38. Tannenbaum, S. R., D. Fett, V. R. Young, P. D. Lang, and W. R. Bruce. 1978. Nitrite and nitrate are formed by endogenous synthesis in the human intestine. Science (Wash. DC). 200(4349):1487-1489.

39. Stamler, J. S., D. J. Singel, and J. Loscalzo. 1992. Biochemistry of nitric oxide and its redox-activated forms. Science (Wash. DC). 258:1898-1902.

40. Jocelyn, P. C. 1972. Thiols and disulfides in blood. In Biochemistry of the SH Group. Academic Press Limited, London. 240-260.

41. Lewis, S. D., D. C. Misra, and J. A. Shafer. 1980. Determination of interactive thiol ionizations in bovine serum albumin, glutathione, and other thiols by potentiometric difference titration. Biochemistry. 19:6129-6137.

42. Mordvintsev, P. I., V. G. Rudneva, A. F. Vanin, L. L. Shimkevitch, and B. I. Khorodov. 1986. Inhibition of platelet aggregation by dinitrosyl iron complexes with low molecular weight ligands. Biokhimya. 51:1851-1857.

43. Malinski, T., and Z. Taha. 1992. Nitric oxide release from a single cell measured in situ by a porphyrinic-based microsensor. Nature (Lond.). 358:676677. 